\title{
Interval timing routines for the IBM PC/XT/AT microcomputer family
}

\author{
MICHAEL BÜHRER, BERND SPARRER, and ROLF WEITKUNAT \\ University of Munich, Munich, Federal Republic of Germany
}

\begin{abstract}
We describe a 1-msec software timer for measuring response latencies and controlling delays on the IBM PC/XT/AT without additional hardware requirements. To demonstrate the machine language routines, a short BASIC example program is included. In a simple experimental design, two different stimulus words are presented on screen and keypress response latencies are measured. Precise timing of stimulus presentation is accomplished by direct manipulation of the video controller. The principles of programming interrupt-controlled timing routines are addressed to be easily adapted to other problems or different programming languages.
\end{abstract}

Designs in experimental behavior research often require exact time-interval measurement and control. Subjects' response latencies or delays of external events often have to be assessed; interstimulus intervals, intertrial intervals, and duration of stimulus presentation need to be timed in order to control the experimental process. Other applications combine two or more of these components (e.g., response latency windows).

Dlhopolsky (1983) proposed two Z-80 machine language millisecond timers that are software designed but based on hardware clock cycles of the TRS-80. Femano and Pfaff (1983) developed an interval handler for the 6502 microprocessor using external circuitry. Although such solutions achieve very high resolutions (less than $50 \mu \mathrm{sec}$ ), they depend on specific hardware requirements.

Currently the standard in microcomputers, in terms of laboratory and analysis applications, tends to be established by the IBM personal computer family. A wide variety of hardware add-ons is available from numerous suppliers. However, adequate add-ons for the laboratory standard of minicomputers (DEC's PDP-11) are unavailable. Particularly, external clocks usually are unable to interrupt the processor, which causes various programming difficulties.

On the other hand, the prerequisites for programming interval timing routines are already built-in features of the IBM PC/XT/AT computer family. In order to demonstrate this, the first part of the present paper describes a simple BASIC program, which makes use of an assembly routine that performs all the difficult steps of timing control. The second part explains the technical details and programming techniques needed to tailor the assembly language routines to the reader's own requirements.

The authors' mailing address is: Institut für Psychologie, Klinische Psychologie, Universität München, Geschwister-Scholl-Platz 1, D-8000 München 22, Federal Republic of Germany.

\section{REACTION-TIME MEASUREMENT}

The BASIC program shown in Listing 1 demonstrates an experimental session in which reaction time to two different verbal stimuli is measured. Such a procedure has to fulfill two critical requirements. Delay and reaction times have to be measured precisely, and the stimulus must be presented at a predetermined point in time. These two requirements are met by an assembly language programmed timer, which is able to measure time intervals with a resolution of $1 \mathrm{msec}$, and a pseudotachistoscopic stimulus presentation. The latter is achieved by switching the video display controller off and moving the contents of a prewritten RAM screen into the display memory area. This program design guarantees exact process control even with complex stimulus patterns.

Before presentation of the stimulus, a warning stimulus is given, defined as the moment when the text on the screen disappears. After a predefined delay, the imperative stimulus is presented. It consists of one of two words appearing centered on the video display. The time interval between the warning stimulus and the imperative stimulus is controlled by the delay timer. When the display is turned on and the stimulus appears, the latency timer starts to measure the time until the subject presses a key. The next trial is delayed by a random interstimulus interval. Each word is presented five times in a pseudorandom sequence. At the end of the session, means and standard deviations of response latencies are computed and displayed.

There is a limitation to the exactness of stimulus presentation using a video display. The minimum time required to make a stimulus pattern visible is determined by the refresh rate of the monitor $(16.7 \mathrm{msec}$ for a $60-\mathrm{Hz}$ monitor). This could be compensated by synchronizing stimulus presentation with vertical retrace of the electron beam. Since not all display adapters provide the necessary system information, this feature is not implemented in the present program, although the assembly routine offers a 
solution to this problem for Color and Hercules Graphics adapters.

The BASIC program is designed for IBM's BASICA interpreter (Version 3.0 or later) or Microsoft's GWBASIC (Version 2.01 or later). The assembly routines should be assembled with Microsoft's MASM (Version 4.0). The program runs on all IBM PC/XT/AT models and on most compatibles. For compatibles, it is a good idea to check the input frequency of the timer/counter chip for substantial deviations from $1.1931817 \mathrm{MHz}$. (Usually, the computer's technical reference manual provides this information.)

Lines $100-200$ initialize all variables used. This is mandatory since interpreter BASIC reorganizes memory when new variables are created. This reorganization must be avoided to ensure that the location of machine language subroutines in memory remains unchanged. Line 160 sets the number of different stimulus words (NWORDS) and the number of presentations of each word (NTRIALS). These values may be changed for other designs (the DATA statements of lines 5030 and 5060 then have to be adjusted). The variable MS in line 140 defines the delay time in milliseconds between the warning and imperative stimulus. It should be long enough to allow for the screen to become completely black. MAXRES defines the maximum response time allowed in milliseconds. This prevents the computer from locking up if the subject fails to respond. The dimension statements in lines 290-310 define the array where the assembly routine will be stored (IASM), a RAM screen (ISCRN) that will be filled with the stimulus text, and the arrays for interstimulus intervals (ISI) and measured latencies (LATB).

Line 320 defines ISUBRT as the starting address of array IASM. ISUBRT will be used to load and call the assembly routine. Loading may be done in two ways: Either subroutine 6000 is called, which reads the DATA statements beginning at line 9000 , pokes the data into IASM, and checks for typing errors, or BLOAD is used to read a binary file (produced by MASM; see Listing 2) from disk. The latter, of course, relieves the user from typing lines 6000 to 9480 .

The interstimulus intervals (ISIs) are random generated in subroutine 2010. First, the minimum and maximum interstimulus intervals are entered in seconds. The minimum must be greater than $3 \mathrm{sec}$, because BASIC fills the RAM screen during the interval (see line 440). Compiled BASIC decreases this limit.

Writing to the RAM screen is accomplished in lines $1020-1120$. First, the whole $80 \times 25$ integer array is filled with blanks, where low bytes contain the ASCII code 20 (hex) and high bytes set the normal screen attribute 07 . Lines 1050-1110 center the stimulus word on the screen.

Line 410 starts the main program loop. The ISI is timed by BASIC's simple TIMER function (lines 420 and 450). Next, subroutine 3010 is called, which calls ISUBRT passing the arguments MS (delay between warning and imperative stimulus), ISCRN (starting address of RAM screen), LAT (on input, LAT contains the value of max- imum response time allowed [MAXRES]; on output, it returns measured response latency), and KEYP (returns ASCII code of key pressed in low byte and keyboard scancode in high byte). In case the subject fails to respond, both output parameters (LAT, KEYP) are set to zero. When ISUBRT returns to BASIC, the main loop continues. Following the last response latency measurement, statistics are calculated and results are presented (lines 4010-4260).

\section{HARDWARE}

The Intel 8253 16-bit timer/counter circuit (located on the motherboard) provides three programmable interval timer channels, of which channel 0 is used to maintain the time of day for the system. The other two channels are reserved for system internal purposes (see Sargent \& Shoemaker, 1984). The input clock frequency for the 8253 is derived from the system clock $(4.772727 / 4=$ $1.1931817 \mathrm{MHz}$ ). The output frequency is determined by a programmable 16-bit counter, which for channel 0 is set to 65536 , that is, $18.2 \mathrm{~Hz}$. Every tick causes a hardware interrupt to channel 0 of the $8259 \mathrm{~A}$ interrupt controller. On system start, the interrupt controller is initialized so that interrupt 8 will keep track of the time of day. This interrupt routine is then redirected to software interrupt 1C (hex). The service routine of interrupt $1 \mathrm{C}$ is only an IRET instruction (return from interrupt) and offers an opportunity to the user to get control of the timer interrupt. IBM advises users to utilize this clock for all timing purposes in order to obtain maximum compatibility with all models of the computer family. The company promises to maintain compatibility in future systems (International Business Machines, 1986).

\section{TIMER}

Listing 2 contains the program listing in assembly language, which demonstrates the programming of delay and response latency routines. The redirection of the timer interrupt is accomplished by subroutine SETINT. (The use of register BX as relocation factor will be explained later.) First, the address of the old interrupt routine is determined and saved in the two variables INTSEG (segment) and INTOFS (offset). Then the interrupt vector is set so that it points to the routine NEWINT, which then handles the timer interrupt. NEWINT simply increments the counter INTCNT every time it is called by the timer interrupt 1c. In order to obtain a higher resolution than the actual $18.2 \mathrm{~Hz}$, FAST changes the programmed value of counter 0 of the 8253 timer/counter chip to 4 A9 (hex). The interrupt frequency is thereby changed to $1000.1522 \mathrm{~Hz}$. The resolution then is $1 \mathrm{msec}$, which is sufficient for most applications. Since an exact frequency of $1 \mathrm{kHz}$ is not programmable, a deviation of $152 \mathrm{nsec}$ per millisecond is accumulated. If the time intervals desired are considerably larger than $1 \mathrm{sec}$, measures should be taken in calling the BASIC program to correct 
for the error. If a different resolution is desired, another counter value can be calculated by the formula Count $=$ INT(1.1931817/TargetFrequency).

The delay function is implemented in the main procedure. The principle is to initialize the counter INTCNT to zero, and to compare the millisecond argument passed by the caller with the contents of INTCNT. If INTCNT is still lower than delay time, a jump back is performed to continue comparison (see label DELAY).

The latency function (following delay) also clears the counter. Then the keyboard buffer is flushed to ensure that no prior keypress is misinterpreted as a valid response. Next, CX is loaded with the argument RESULT, which on input contains the maximum response time allowed in milliseconds. Then the latency loop is entered, which first checks if maximum response time is exceeded. If so, the loop is terminated, and parameters RESULT and KEY are set to zero. Otherwise, software interrupt 16 (hex) is called. Function 1 of interrupt 16 sets the zero flag if no character is typed. When a keypress (i.e., subject's response) occurs, the actual value of INTCNT is written to the argument RESULT. Any other input source (e.g., Centronics, game ports, or digital I/O devices) can be used to substitute for this method, which might be inappropriate for some applications.

The maximum time period that can be used in the delay and latency routines is $65,535 \mathrm{msec}$ (values must be passed in "two's complement" coding, where numbers above 32767 are represented as negative numbers: IF $x>32767$ THEN $x=x-65536$ ). This also holds for maximum response time allowed.

Changing the timer interrupt frequency results in the side effect that the system's time of day is lost. After the program is run, the time should be updated by polling the battery clock.

\section{ADDITIONAL ASSEMBLY FEATURES}

To present a warning stimulus, the video controller is disabled by clearing the video enable bit of the CRT mode register that blanks the screen (CRT_DISABLE). Thereby, it is possible to change the contents of the video memory without any visible changes on the display. At this point in the program flow, it is important to clear the delay counter, because the timer interrupt already increments the counter every millisecond. During delay timing the assembly routine swaps the RAM screen to the location of the video screen (SWPSCRN). SWPSCRN determines the kind of display adapter used in the system and copies the RAM area to the display area. Since the starting address of the RAM area is passed as an argument, it is possible to define different RAM screens in the BASIC program and use them as imperative stimuli. When delay has finished, the display is enabled again (CRT_ENABLE) and the latency counter is cleared.
From that point, the interrupt routine measures the response latency.

In order to tie response latency measurement to the vertical retrace of the video controller, routine CRT ENABLE contains a commented area of code, which can be included in the program by deleting the comment signs. This piece of code will wait for the vertical retrace bit to indicate the moment of synchronization. From then on latency will be measured. This feature is not available for the IBM monochrome display adapter, which does not provide this information. Since Hercules Monochrome Graphics Cards use a different port and logic, the code for both Hercules and Color Graphics adapters is included.

Whereas implementing this synchronization suppresses additional artificial variance in the response latency data, another constant bias should be taken into account. The time elapsed from starting the counter to the actual presentation depends on the vertical screen position of the stimulus. This can be easily corrected by the formula:

time $=y \_$position $*((1000 /$ monitor_refresh_rate $) /$ No_of _lines $)$.

Multilanguage programming from BASIC requires the following considerations: (1) When loaded by the BLOAD command, a binary file must have a BSAVE header (see BS_Hdr, Listing 2), which contains the identification (OFD hex), four unused bytes, and the module size in bytes. Module size is calculated by the assembler using the HEADER and TRAILER locations. The 7-byte BSAVE header will be the first in the binary file and must be removed when DATA statements are generated. (2) Assembly routines callable from BASIC must be relocatable. This requirement precludes the declaration of variables inside the routines. In order to handle this restriction, the procedure RELOC calculates a relocation factor, which is the offset to BASIC's code segment, and returns it in BX. BX then points to the beginning of the assembly routine. At the end of the listing, three variables are defined. Whenever these variables are addressed, BX is added to the offset of the variables. (This routine assumes that CS and DS point to the same segment.)

\section{REFERENCES}

DLhopolsky, J. G. (1983). Machine language millisecond timers for the Z-80 microprocessor. Behavior Research Methods \& Instrumentation, 15, 511-520.

Femano, P. A., \& Pfaff, D. W. (1983). Time-interval acquisition on a 6502-based microcomputer. Behavior Research Methods \& Instrumentation, 15, 521-529.

INTERNATIONAL BuSINESS MACHINES (1986). Technical reference (IBM Personal Computer AT 6183355). Boca Raton, FL: Author.

Sargent, M., III, \& Shoemaker, R. L. (1984). The IBM personal computer from the inside out. Reading, MA: Addison-Wesley.

(Manuscript received October 31, 1986; revision accepted for publication January 30, 1987.) 
LISTING 1

BASIC Demonstration Program

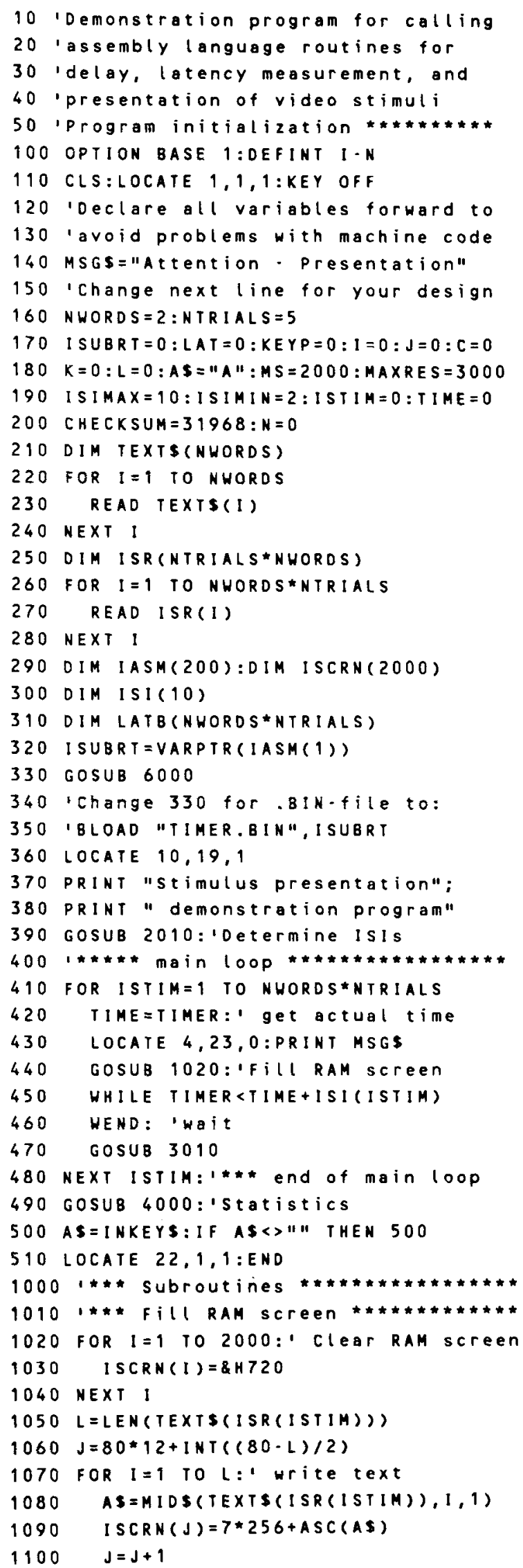


LISTING 1 (Continued)

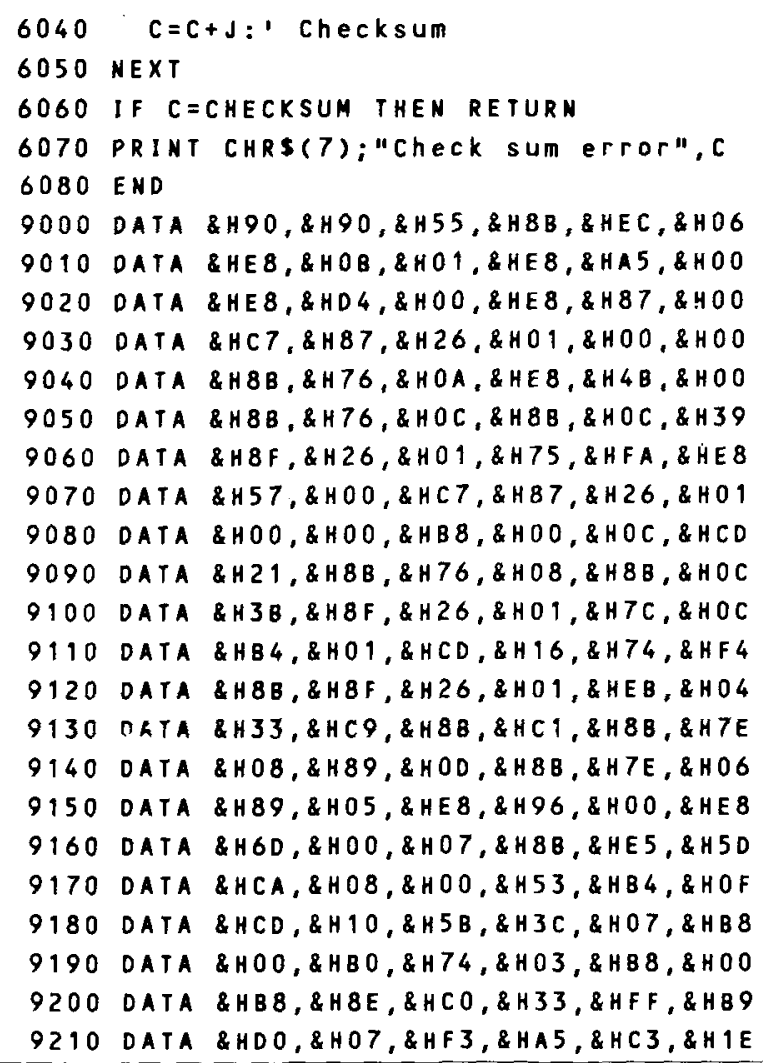

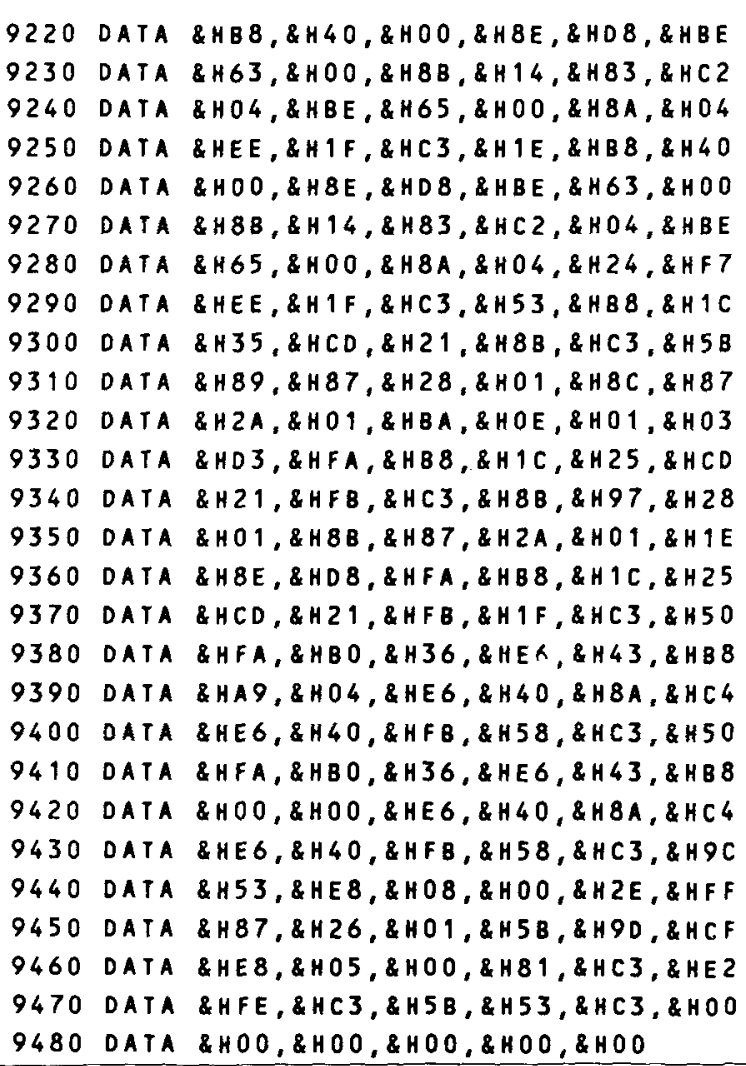

\section{LISTING 2}

Assembly Language Timing Routine

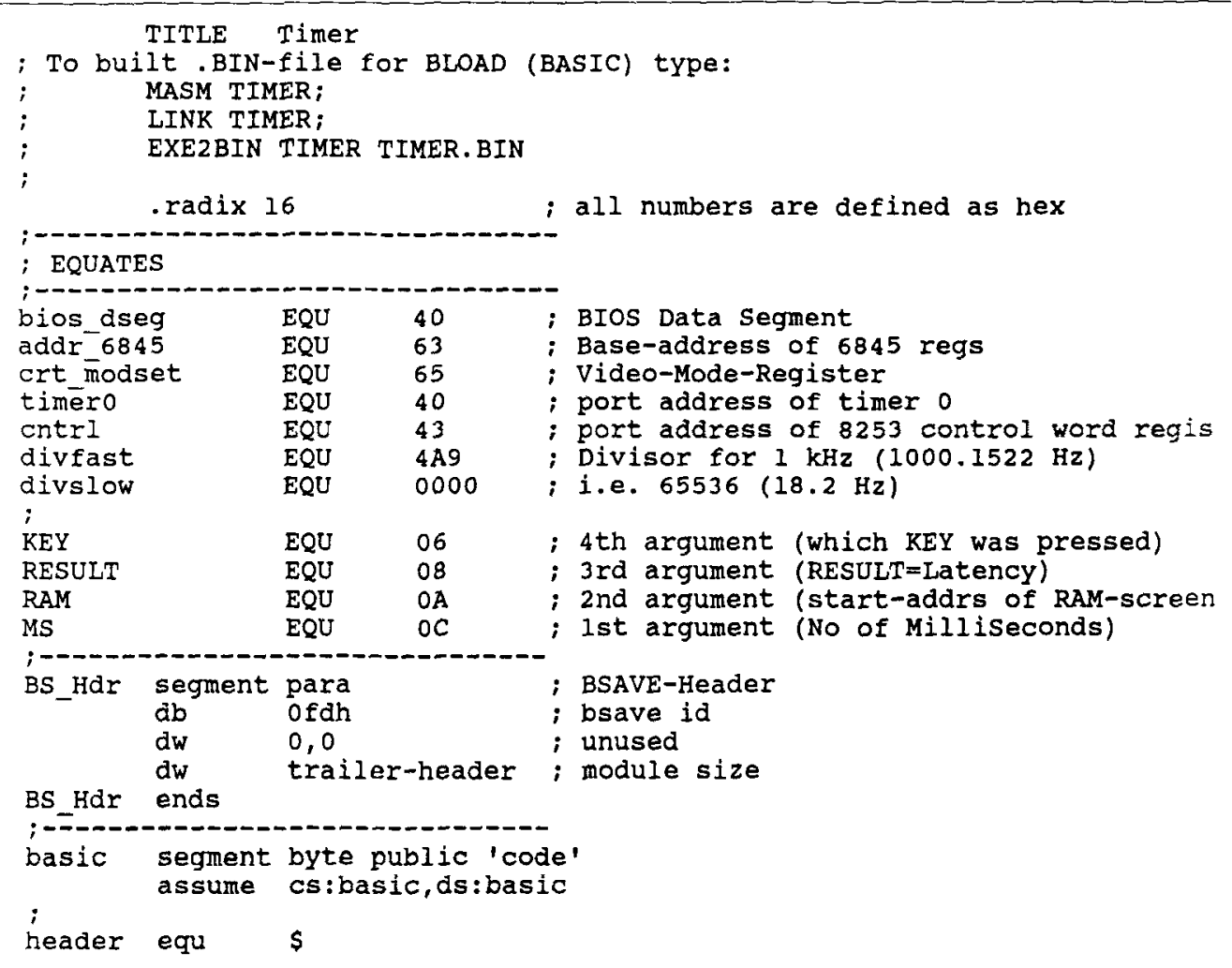


LISTING 2 (Continued)

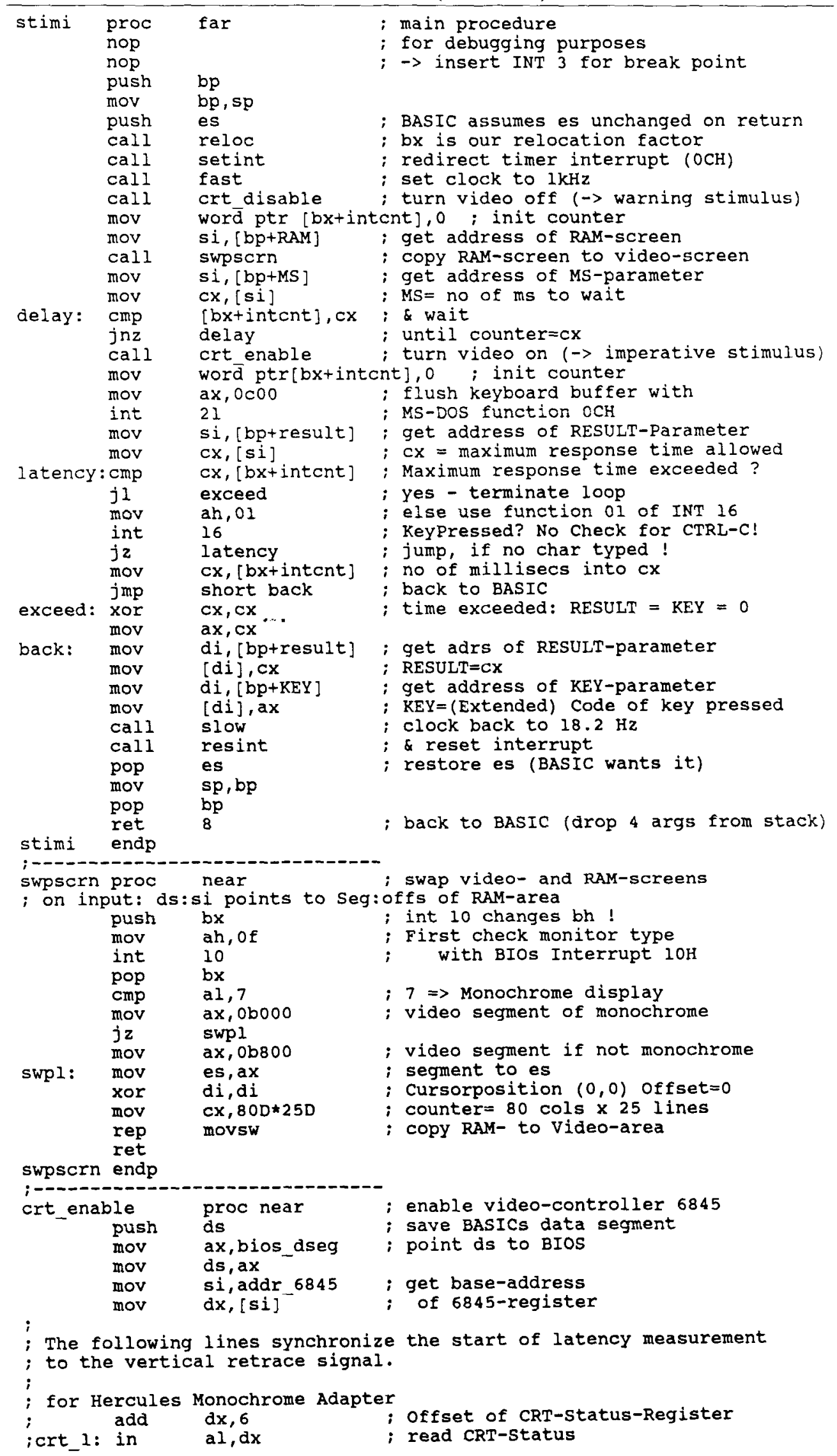


LISTING 2 (Continued)

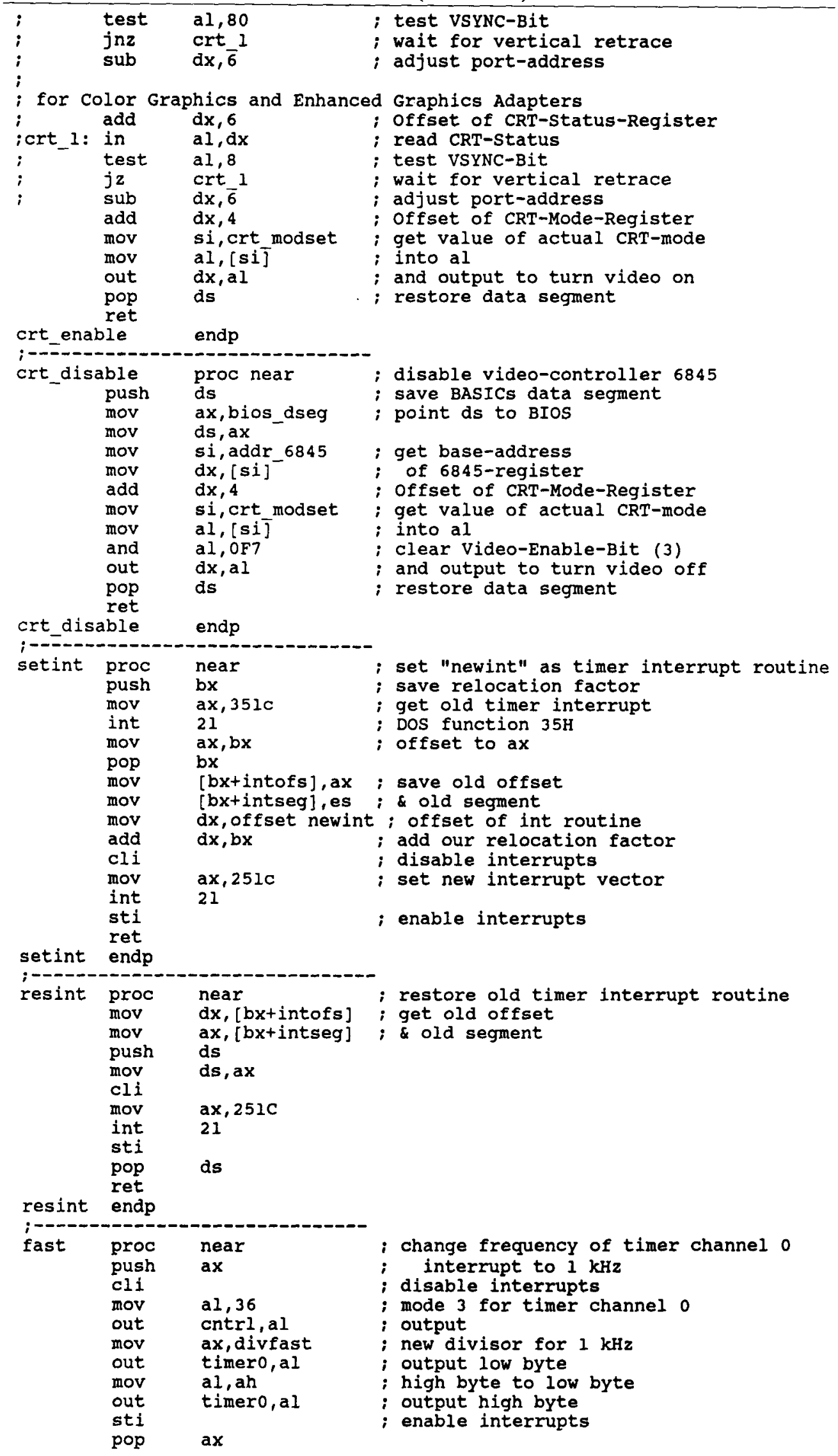


LISTING 2 (Continued)

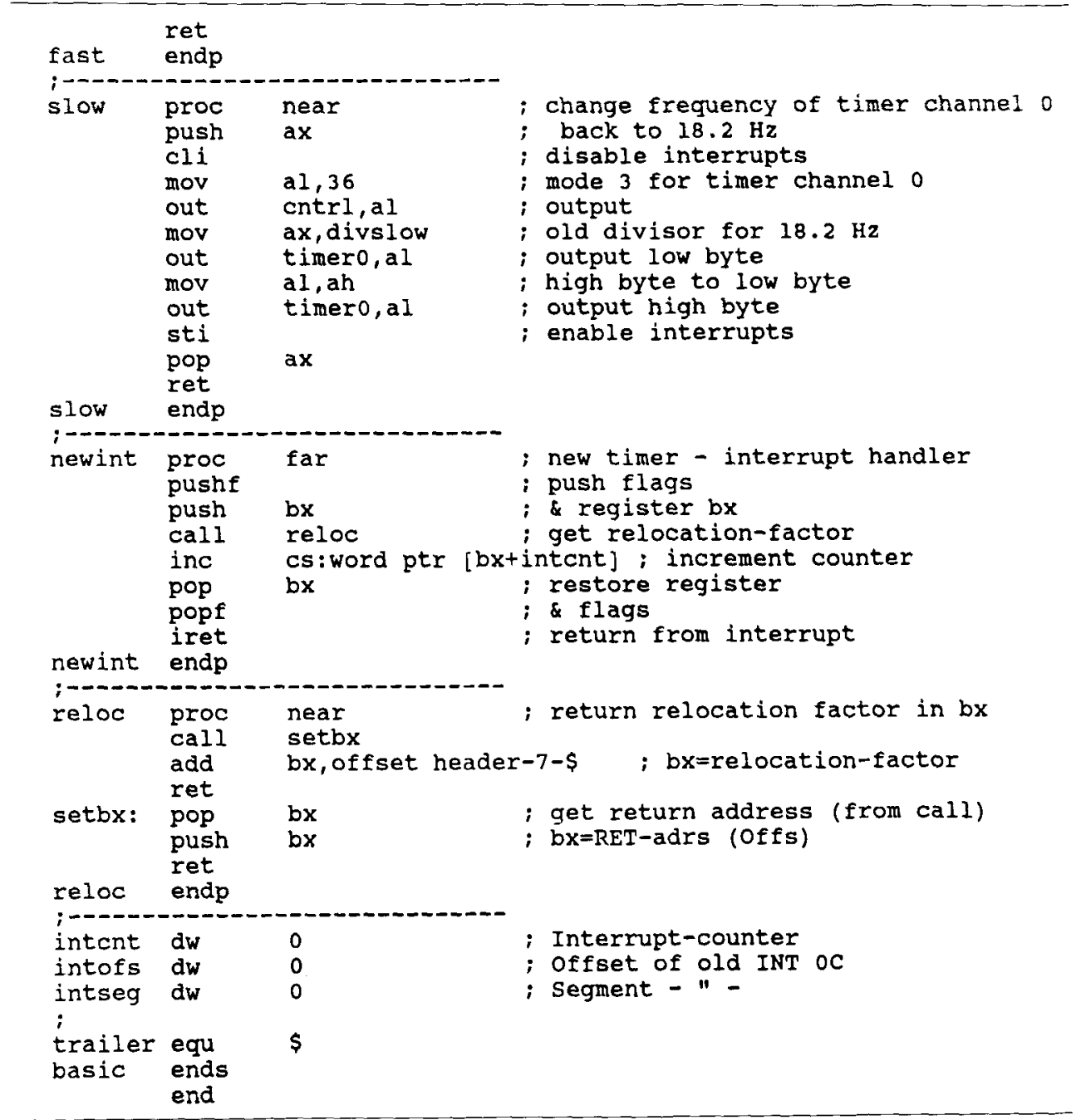

(Manuscript received October 31, 1986; revision accepted for publication January $30,1987$. ) 